Original Research Paper

\title{
The Architecture of Fuzzy Agent in Rescuer Monitoring System
}

\author{
Azwin Hazrina Ahmad, Ahmad Kamil Mahmood and Jafreezal Jaafar \\ Department of Computer and Information Sciences, \\ Universiti Teknologi PETRONAS, Bandar Seri Iskandar, 31750 Tronoh, Perak, Malaysia
}

\author{
Article history \\ Received: 26-06-2014 \\ Revised: 21-10-2014 \\ Accepted: 30-05-2015 \\ Corresponding Author: \\ Azwin Hazrina Ahmad \\ Department of Computer and \\ Information Sciences, Universiti \\ Teknologi PETRONAS, Bandar \\ Seri Iskandar, 31750 Tronoh, \\ Perak, Malaysia \\ Email: azwinhazrina@yahoo.com
}

\begin{abstract}
Malaysia is a developing country and currently experiences a new emerging safety issue, which considered as critical. Country likes Malaysia is in aggressive mission to develop its country by year 2020. In the effort to achieve developed country, it requires development in each industry. There will be more works should be done by workers in the same duration compares to the last few years. With lots of tasks, people get stress out and this will lead to mistakes. Unsafe action sometimes cause accidents, worst may cause death. Especially hazardous works such as professional that need to work in manhole, low oxygen level confined space, underwater and fire fighter. This people need to be monitored during their operation to ensure their safety, thus the operation can complete successfully. In this study, we focus on the personal monitoring for fire fighter during their rescue operation. An agent based monitoring system using type-1 fuzzy logic is simulated to see the effect on personal's parameters, such as breathing rate, breathing air level and body motion. A case study has been done to get the real fuzzy data which gathered by sensors to be tested using Mamdani type-1 fuzzy method. The result produced a reliable crisp output which then processed by the system to alerts the fire fighter any predicted accident that might happen. Intelligent agent adopted in this engine helps monitoring system act autonomously and process a reliable decision. This engine generate precise and reliable output from fuzzy input as we deal with human lives. As the result, injuries or fatality during works can be reduced.
\end{abstract}

Keywords: Personal Monitoring, Self-Contain Breathing Apparatus (SCBA), Self-Contain Underwater Breathing Apparatus (SCUBA), Permanent Disability (PD)

\section{Introduction}

Personal safety is very important for individual whose work is classified as dangerous. The example of hazardous works, such as being a fire fighter who needs to rescue victims in fire, sewerage professional who need to fix problem in manhole, engineers checks the underground electric generation plant, SCUBA rescuer. These professional, when enter their work place, they need to wear an apparatus called SelfContain Breathing Apparatus (SCBA) or Self-Contain Underwater Breathing Apparatus (SCUBA). This apparatus complete with Breathing Rate Sensor, Tank Breathing Air Level Sensor and Motion Sensor. The main problem of current personal monitoring system is-they use manual system. Where each person who's instructed to enter the fire, confine space or manhole, they just get a safety briefing before their operation.
Throughout the operation, there is no monitoring of each person status.

There are many reasons why reliable monitoring system is crucial for safety during works, example:

- If two persons get into hazardous work place, manual system only can monitor or communicate with one person at one time and only depends on their walkie-talkie. While, this newly designed system can monitor more than one person at one time by viewing the status of their Breathing Rate data, Tank Breathing Air data and Body Motion data at display panel

- If one of them collapses during the rescue operation and used only walkie-talkie, instruction and actions should be done usually delayed due to uncertainties in human language. While, type-2 fuzzy agent monitoring system provides real time 
personal status monitoring complete with automatic alarm signal as 'red alert

- If one of them collapse or missing during operation, manual system will ask another fire fighter to seek the collapse fire fighter. This will need for manual communication and decision making by authorized expert which lead to delay in rescuing work. In type- 2 fuzzy agent personal monitoring system, intelligent calculation used in order to make critical decisions and process automatic alarm signal to dedicated fire fighter

- Human language consists of uncertain information. If it used as input in critical situation and did not correctly processed, the output will be imprecise and may cause wrong decision and actions

- Modeling the wrong input and output will be the cause of unsafe act and unsafe condition. This might cause harm to fire fighter, who's performing rescue works in hazardous environment. Worst may cause fatalities

From local statistic regarding accident happen during works, which caused Non-Permanent Disability (NPD), Permanent Disability (PD) and Death (D) to the workers can be view in graphs below (Fig. 1-6).

From the statistic shown, the major problem of human disabilities and fatalities happen during work must be overcome. The number stated in the graphs are all defines human lives. It is crucial to save our man from predicted accident during works rather than cure or we lose our trained professionals. The main problem persist now, professionals has not been monitored at real time manner from the start of their operation until finish. Furthermore, traditional monitoring sometimes fail due to uncertain language used during emergency while they are working. The uncertain words may cause uncertain act and decision produced.

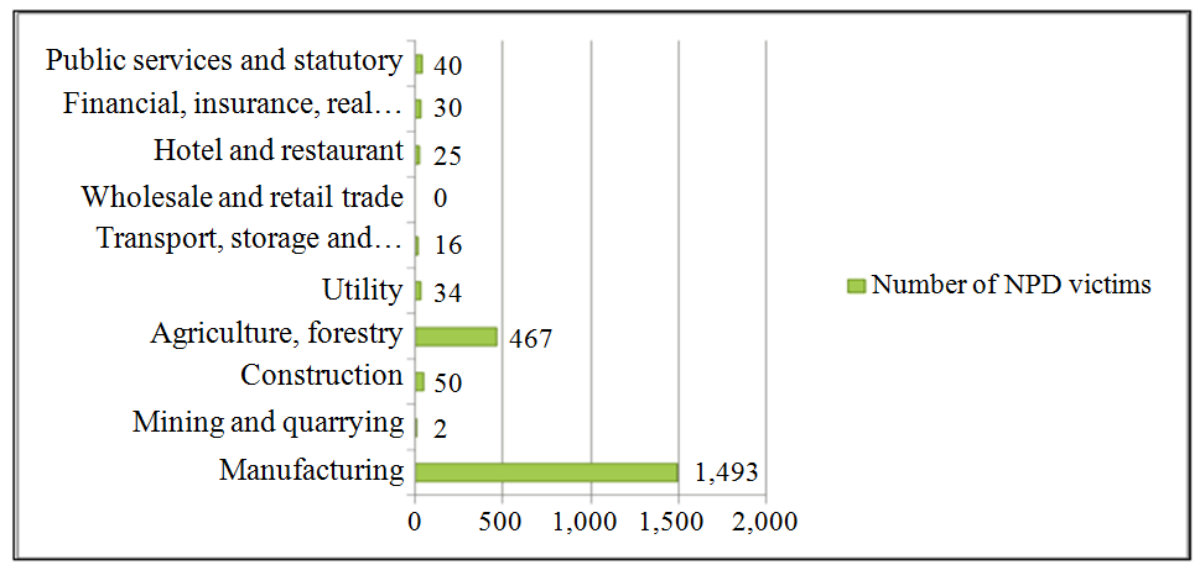

Fig. 1. Number of investigated Non-Permanent Disability (NPD) victims during works in Malaysia (Year 2010)

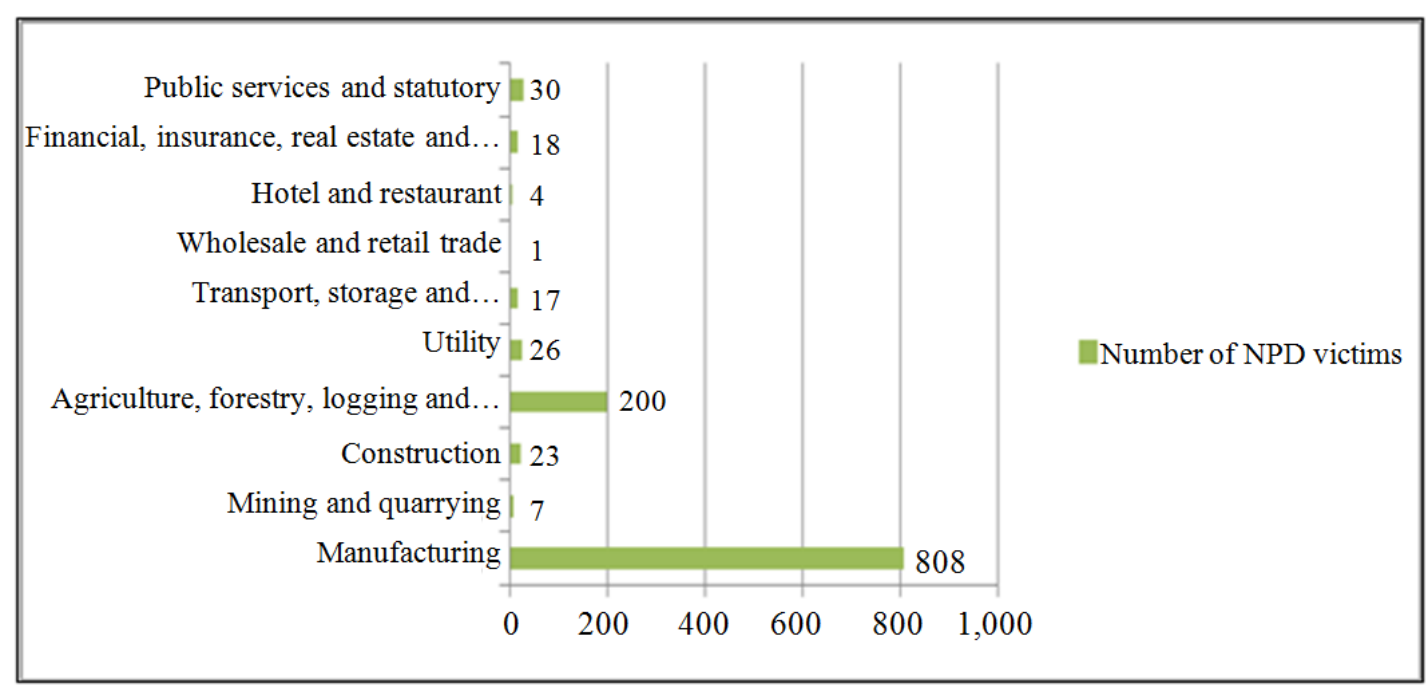

Fig. 2. Number of investigated Non-Permanent Disability (NPD) victims during works in Malaysia (Year 2011) 


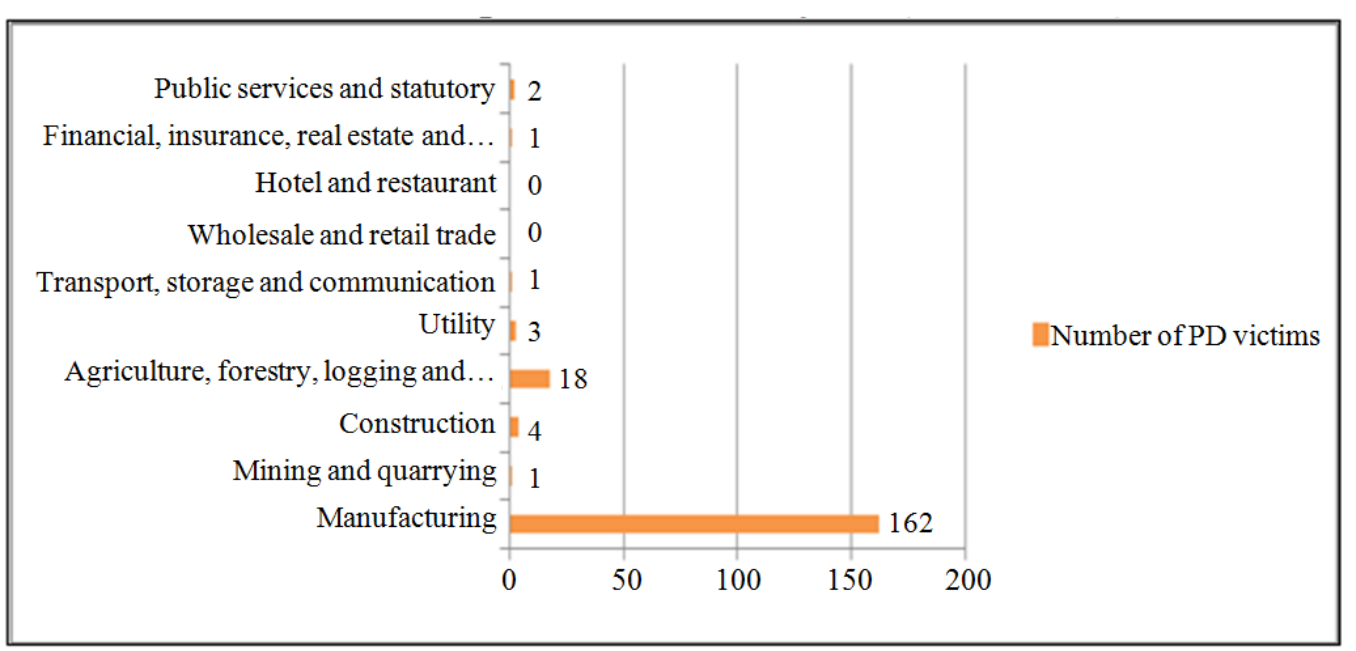

Fig. 3. Number of investigated Permanent Disability (PD) victims during works in Malaysia (Year 2010)

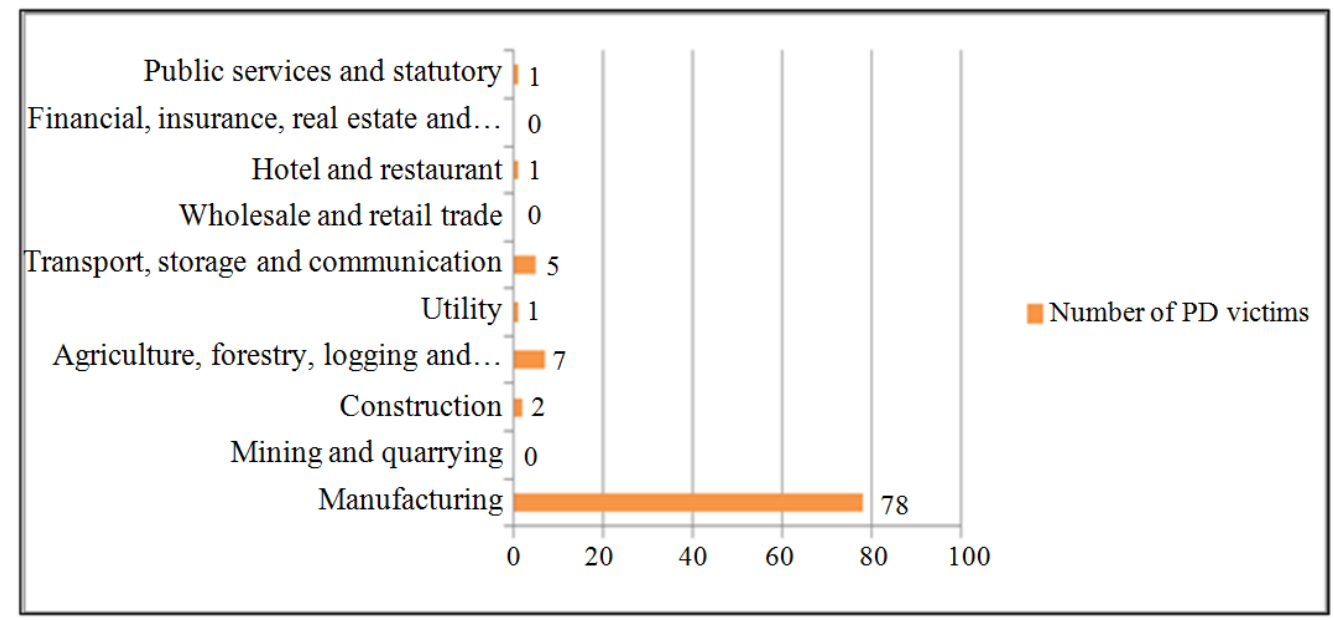

Fig. 4. Number of investigated Permanent Disability (PD) victims during works in Malaysia (Year 2011)

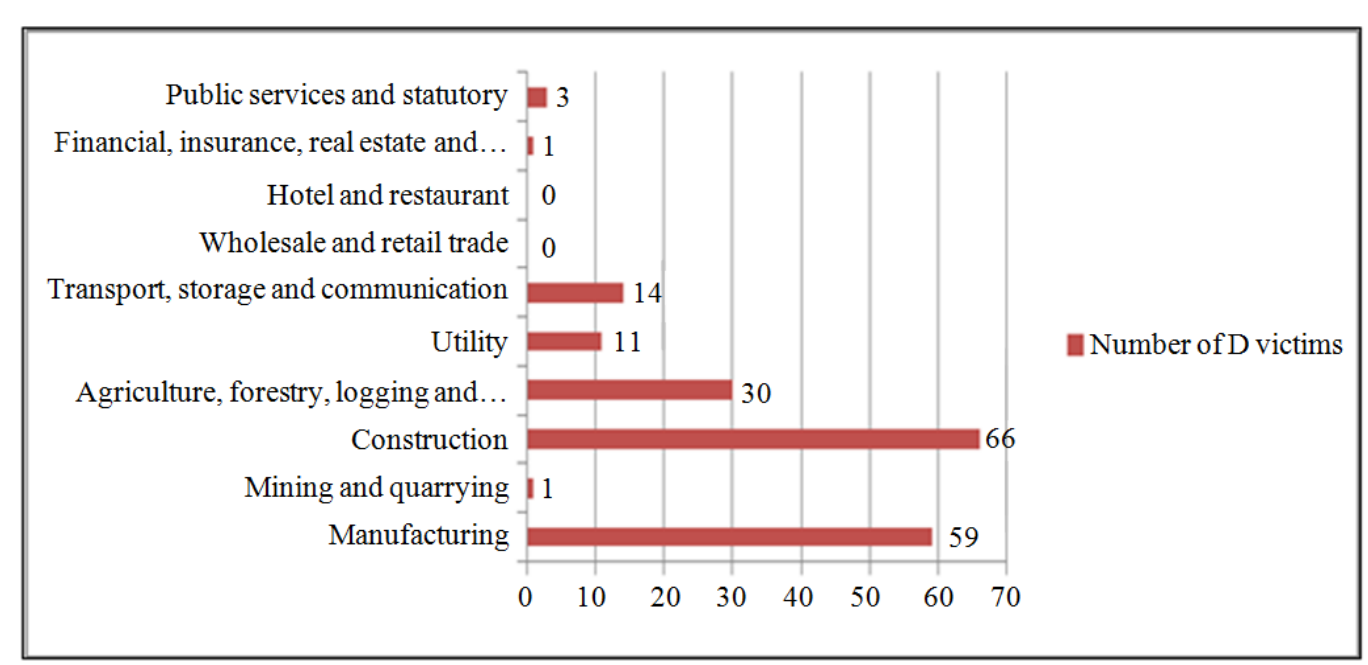

Fig. 5. Number of investigated Death (D) victims during works in Malaysia (Year 2010) 


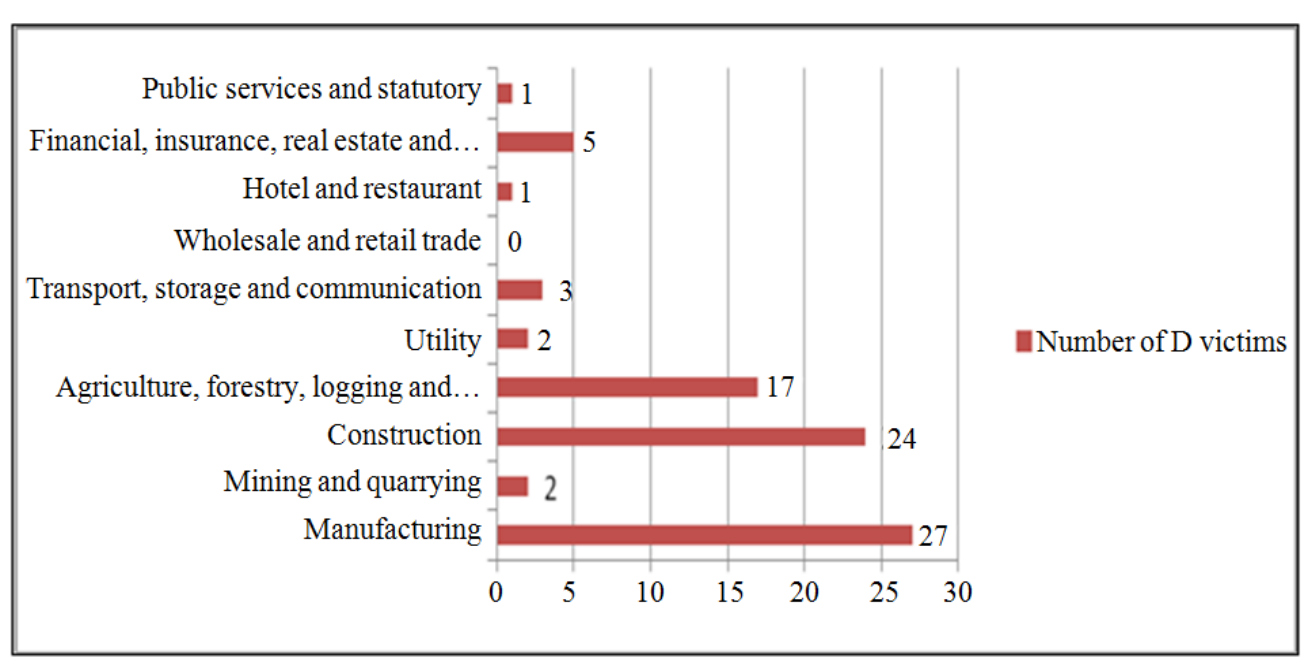

Fig. 6. Number of Investigated Death (D) Victims during Works in Malaysia (Year 2011)

Objective of the study is to formulate new types of agent for personal monitoring system using type-1 fuzzy logic. With the outlined architecture of type-1 fuzzy agent, we hope that the problems defined in current monitoring system can be upgraded, thus reduce the number of human accidents while performing their work.

\section{Background}

The emerging of safety industry in modernization processes needs of technology as tool to solve complex engineering system. Artificial intelligent has offers several method to solve those complex engineering system, such as, expert system, genetic algorithm, neural network and software agent. In this research we combine two main methods in order to solve this issue; they are intelligent agent and type-2 fuzzy logic.

\section{Intelligent Agent}

In this research, we use agent to be the monitoring system engine. What is agent? Nwana and Ndumu classified the agent technology into seven types. They are Collaborative Agents, Interface Agents, Mobile Agents, Information/Internet Agents, Reactive Agents, Hybrid Agents and Smart Agents (Nwana and Ndumu, 1997). The concept of agent stems from the lexicon of Distributed Artificial Intelligence (DAI) popular in 1970s (Paolucci and Sacile, 2005). Agents represent the real relationship between system and the stakeholders. Now, the agent technology has developed and we choose the intelligent agent to be built together with type- 2 fuzzy algorithm.

What's make intelligent agent different from other agents? Intelligent agent defined by Padgham et al., (2005) as a piece of software that is situated (exist in an environment), autonomous (independent, not controlled externally), reactive (responds in a timely manner to changes in its environment), proactive (persistently pursue goals), flexible (has multiple ways of achieving goals), robust (recovers from failure) and social (interacts with other agents) (Padgham and Winikoff, 2005). Intelligent agent suggested by Wooldridge (2009) as manner of reactivity, proactivity and sociality. Intelligent agent must be reactive in perceive and respond to environment changes, proactive to exhibit goal-directed behavior by taking the initiative and has social ability which defines the capability of agent to interact with other agents or human in order to meet its design objectives (Wooldridge, 2009).

\section{Type-1 Fuzzy Logic}

Fuzzy logic theory are theories which define natural phenomenon of the imprecision and uncertainty in most of the system in real world.

Type-1 fuzzy logic is the basic principle of fuzzy logic. Fuzzy logic was introduced by Prof. Lofti A. Zadeh in year 1965. A type-1 fuzzy set is defined in (1) and (2):

$$
\begin{aligned}
& \mu_{A}(x): X \rightarrow[0,1] \\
& \tilde{A}=\left\{\left((x, u), \mu_{\tilde{A}}(x, u)\right) \quad \forall x \in X, \forall u \in J, \subseteq[0,1]\right\}
\end{aligned}
$$

\section{Materials and Methods}

Methodology used as guideline to design and develop a dedicated intelligent agent system. This type-1 fuzzy based personal monitoring system designed and guided by Prometheus methodology. It is an interactive methodology. Prometheus methodology provides "start-to-end" design process, which enhanced by a tool called Prometheus Design Tool (PDT). Furthermore, if we used PDT, it helps in generating basic programming lines in JAVA for 
development pahse. Prometheus has hierarchical designing processes, which allows design to be performed at multiple levels of abstraction. Another advantage of using PDT, it provides automatic crosschecking for our system design. Prometheus supports the internal design of intelligent agent and that makes it suitable for this research where type-2 fuzzy algorithm embedded in the intelligent system.

Using Prometheus methodology will consist of 3 main phases (Fig. 7). They are system specification phase, architectural design phase and detailed design phase. During system specification phase, it is where we do planning and system requirement gathered from the system stakeholder. All of the functionalities of the system are listed with its inputs (percepts) and outputs (actions). Next, the output from the first phase will be used for architectural design phase to determine the agents and interaction between specified agents. Finally the detailed design phase focus on the agent's internal and how it accomplished its tasks within the overall system.

System specification process, consists of:

- Identification of actors and their interactions with the system, in the form of percepts and actions

- Developing scenarios illustrating the system's operation

- Identification of the system goals and sub-goals

- Identifying any external data
- Grouping goals and other items into the basic roles of the system (Paolucci and Sacile, 2005)

The architectural design phase includes three main process, they are:

- Deciding what agent types will be implemented and developing the agent descriptors

- Capturing the system's overall (static) structure using the system overview diagram

- Describing the dynamic behavior of the system using interaction diagram and interaction protocols. (Paolucci and Sacile, 2005)

The detailed design phase includes the following:

- Agent overview to show all of the messages, percepts and action within an agent

- Capability overview to show the data of an agent

- Built in type-2 fuzzy in a selected agent

\section{The Architecture of type-1 Fuzzy Agent}

Fuzzy logic has been a proven problem solving method in the past few years. It provides a simple and easy way to draw a definite conclusion from ambiguous, imprecise or vague information (Khuen et al., 2005). The personal monitoring system suggested in this study can be pictured in Fig. 8 .

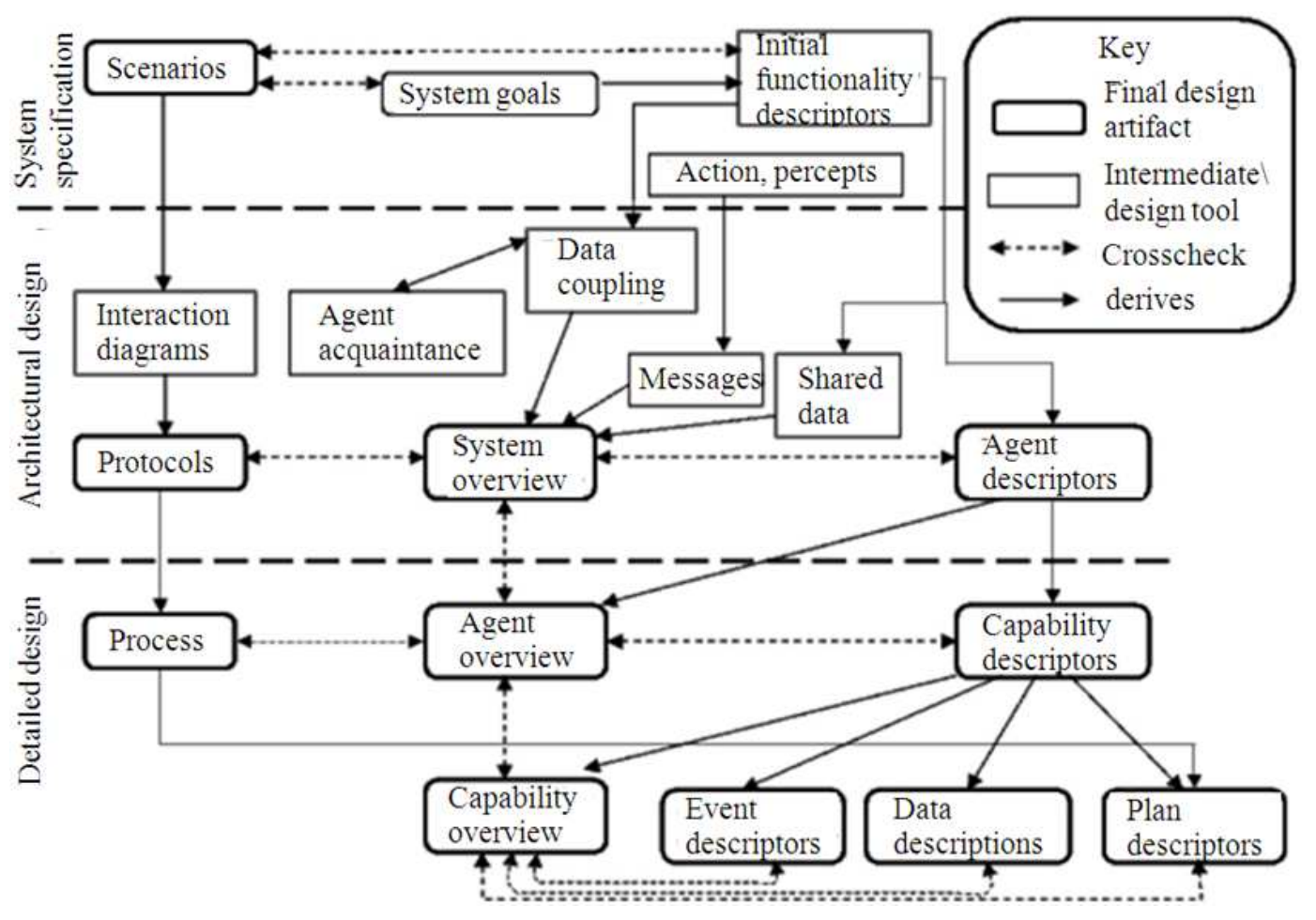

Fig. 7. Phases in Prometheus methodology 


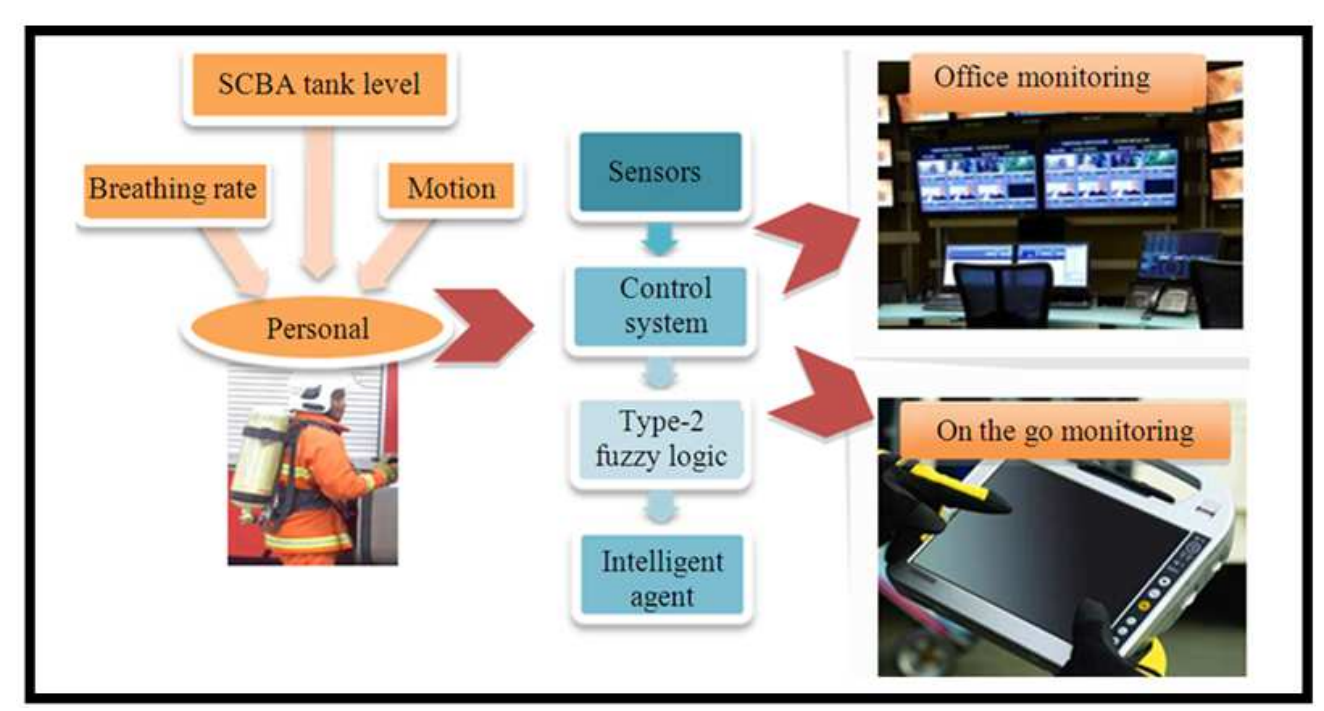

Fig. 8. Personal monitoring system framework

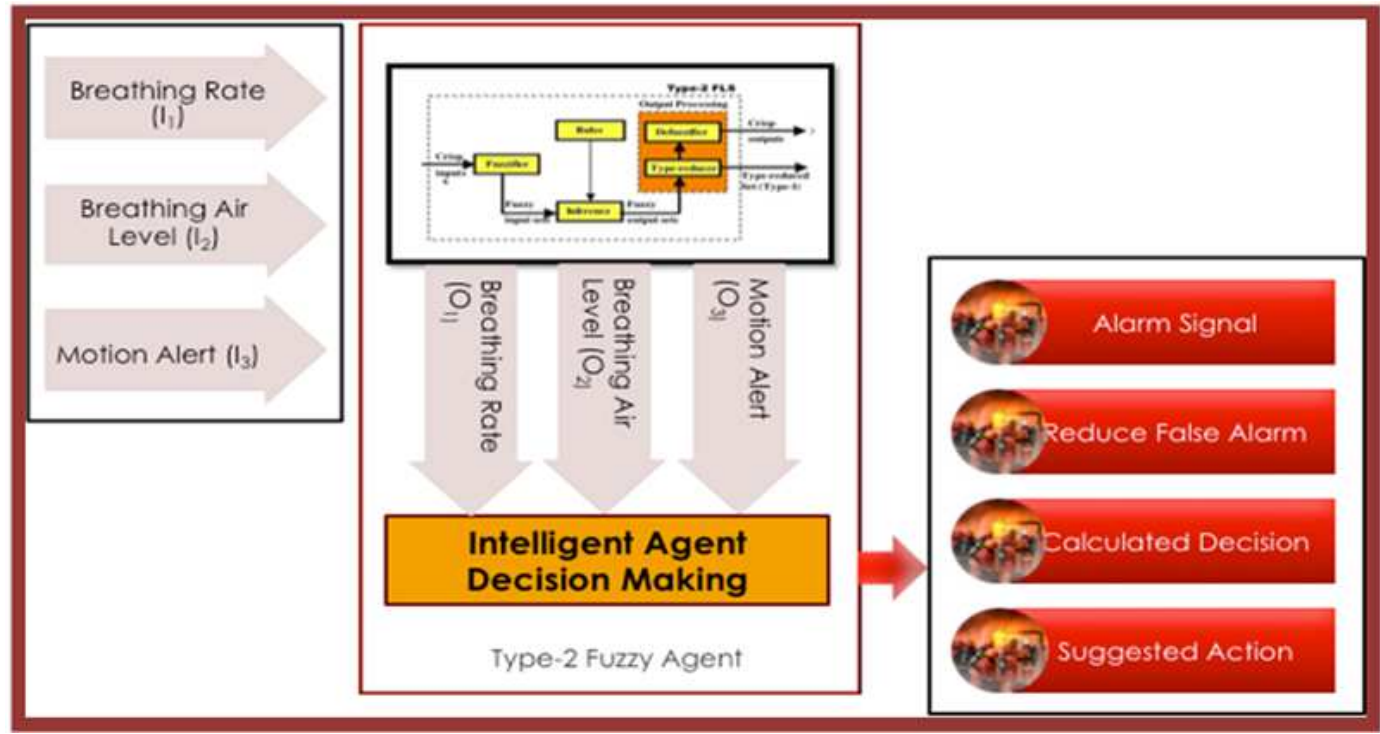

Fig. 9. Architecture of Type-1 Fuzzy Agent Monitoring Engine

In the domain of personal monitoring system for hazardous work, there are lots of input involve such as professional's breathing rate, SCBA breathing Air Tank Level, Professional's Motion, environment pressure, surrounding gas. But in this study, we only focus on three inputs gather from sensors (sensor agent), they are:

- Personal's Breathing Rate

- Personal's Breathing Air Tank's Level

- Personal's Body Motion

While in Fig. 9 below, you can see the type-1 fuzzy agent architecture workflow.

\section{Fuzzy Logic Method}

All of the physical variables above $\left(I_{1}, I_{2}, I_{3}\right)$ is very important to determine our monitored professional status at every time during their operation. So, time (y) will be another input variable in this case. So, let y be the diagnostic rules. Sensors, in other word, Sensor Agent, can identify three linguistic values (low, normal, high) for the three physical variables. $\mathrm{L}_{\mathrm{i}}$ will represent Low Range set. $\mathrm{N}_{\mathrm{i}}$ will represent Normal range set and $\mathrm{H}_{\mathrm{i}}$ will represent High range set for input data, $\mathrm{xn}$ where $\mathrm{n}=1,2$ or 3 . Hence, cases will be symbolized by $\mathrm{C}_{1}, \mathrm{C}_{2}, \mathrm{C}_{3}, \ldots$, cases are based on the knowledge shared by the domain experts 
in real world (Chief of Operation, in the hazadous work domain). Fuzzy Rules can be writing as below:

$$
\begin{aligned}
R(0)= & I F I_{1} \text { is } N_{1} \text { and } I_{2} \text { is } N_{2} \text { and } I_{3} \text { is } N_{3} \text { THEN y i } \\
& C_{1} \ldots \\
R(m)= & \text { IF } I_{n} \text { is } N_{n} \text { and } I_{n}+1 \text { is } N_{n+1} \text { and } I_{n+2} \text { is } N_{n+2} \\
& \text { THEN y is } C_{m}
\end{aligned}
$$

\section{Results}

Type-1 fuzzy logic was used to simulate the rules produced based on expert knowledge, in this case, it was the professional fire rescue team members. The overall risk level of each personal will be determined by three inputs specified, the breathing rate, tank level and body motion. Matlab was used to analysed the range of three main cases of standing, walking (2.5 miles per hour) and running in 5 miles per hour. The result generated by applying Mamdani Type-1 fuzzy logic are shown in Fig. 10-12.

In Fig. 10, the risk-level of each rescuer was determined by the tank-level, breathing-rate and body- motion while they are standing. In Fig. 11, the risk level of walking rescuer within 2.5 miles per hour was presented also based on their tank-level, breathing-rate and body-motion. In Fig. 12, shows the risk level of rescuer whose running within 5 miles per hour. Standing or Case 1 becomes the benchmark for the three cases because the surface is smoother compare to Case 2 and Case 3. In Case 1, rescuer usually in stable condition and does not panic. While in Case 2, rescuer already walk within $2.5 \mathrm{mph}$ and their breathing-rate become higher than Case 1, but still, there is no obvious changes in tank-level and body-motion.

In Case 3, rescuer's breathing-rate, tank-level and body-motion although the surface quite the same in Case 2 , but the normal range for breathing-rate is smaller than range in Case 2. This is the sign where normal breathingrate of human in running condition is small, usually during running; it is hard to get a normal breathing-rate range. Worst when rescuer works in fire scene which consider as extreme environment. In such scene, people will easily get panic and exhausted.

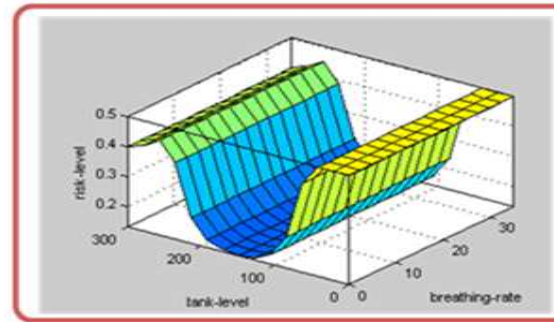

(a)

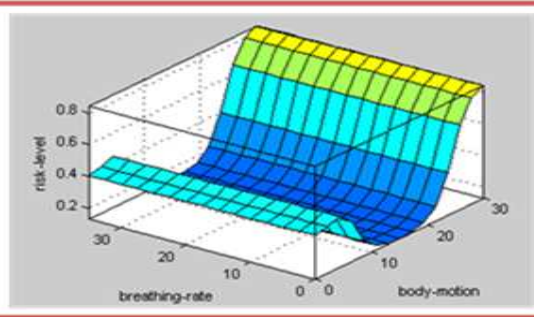

(b)

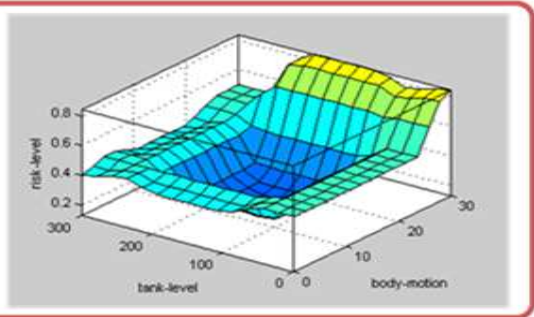

(c)

Fig. 10. Result of type-1 fuzzy agent (Case 1: Standing)

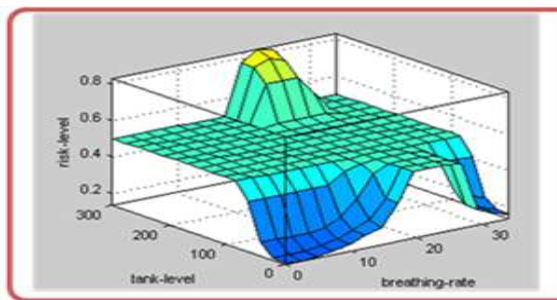

(a)

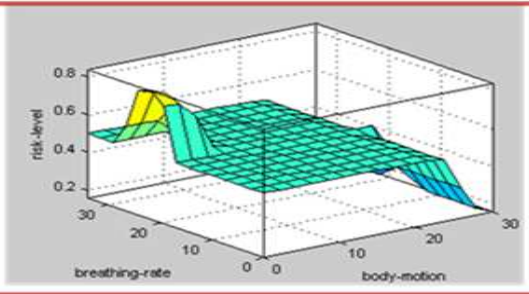

(b)

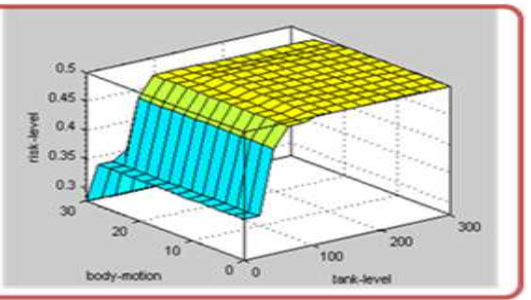

(c)

Fig. 11. Result of type-1 fuzzy agent (Case 2: Walking $2.5 \mathrm{mph}$ )

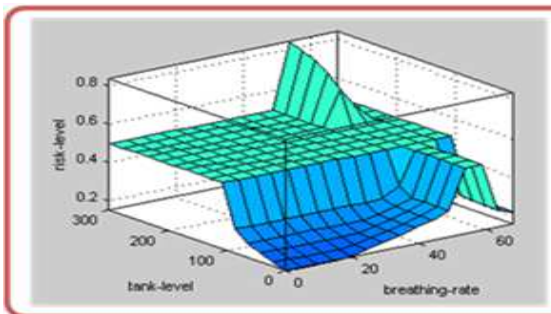

(a)

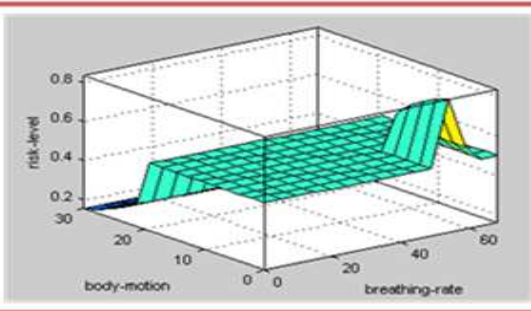

(b)

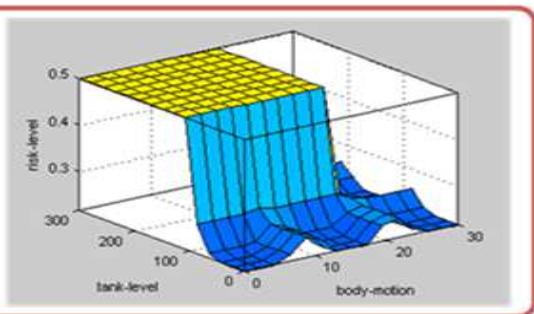

(c)

Fig. 12. Result of type-1 fuzzy agent (Case 3: Running 5 mph) 
Minimum risk-level will only supported with a Medium to High range of tank-level and Normal breathing-rate. In Fig. 10a, Low risk-level of rescuer only supported during Normal breathing-rate and body-motion does not exceed $20 \mathrm{sec}$. In Fig 12c, for Standing rescuer, his risk-level will be at red alert when the tank-level reach below 100 bar and his body-motion reaching $30 \mathrm{sec}$.

Compare to Walking within $2.5 \mathrm{mph}$ case and Running (5 mph) case, as pictured in Fig. 11a-c and 12a-c, rescuer risk-level will determined by at least two inputs status. Such as, (tank-level AND breathing-rate), (breathing-rate AND body-motion) or (tank-level AND body-motion). From these three cases, the best combination of inputs in context of reliable personal monitoring is by using the breathing-rate and body-motion data.

\section{Discussion}

A personal monitoring system using type-1 fuzzy agent is designed to replace manual monitoring system, which does not ensure the safety of workers during their operation. So, we design an architecture of intelligent agent embed with type-1 fuzzy algorithm to handle monitoring problem and calculate the forecast acceptable solutions for the incidents that might happen during the hazardous operation. Results that we have found out from this architecture are:

- Uncertainty handling, where the decision (risklevel) calculation used the uncertain input gathered from sensors can be changed to a TRUE decision. This is the function of type-1 fuzzy logic embedded in the system

- Reduce human expert dependencies, whereusing this architecture of robust and reliable personal monitoring system, will optimize the monitoring rate at every minutes and to the entire rescuer

\section{Conclusion}

In this study, an intelligent agent embedded with type-1 fuzzy characteristic is able to monitor the status of person throughout their operation hours. This hybrid architecture also has special ability to calculate the fuzzy input gathered from sensors and produce the wise decision, learn from human expert knowledge. Intelligent agents have been proposed in this study due to its characteristic advantage to recognize and filter the input and output to achieve the optimum monitoring system operation.

For future study, we suggest that this study extended to a type-2 fuzzy logic, discussed by Bustince et al. (2007) to produce a type-2 fuzzy agent. The new type-2 fuzzy (Castillo and Melin, 2008)) agent can then be applied in other domain such as in Medical industry, Maritime, Governance Surveillance, Oil and
Gas industry. Encourage to gives more inputs in order to make the decision more precise and reliable for personal monitoring, such as, human heart's beat, body temperature and lots more.

\section{Acknowledgment}

I would like to acknowledge Universiti Teknologi PETRONAS for Short Term Internal Research Fund (STIRF) awarded to this project on January 2011. This project also Submitted for Pre Commercialization Fund from Ministry of Science, Technology and Innovation (MOSTI) with the collaboration of industry entity, Semarak Motivasi Sdn Bhd and Universiti Teknologi PETRONAS, as institute of higher learning. Also thanks to Fire and Rescue Department, Malaysia (BOMBA) of Perak Branch due to their support during our case study at the department.

\section{Author's Contributions}

Azwin Hazrina Ahmad: Participated in all experiments, conduct the case study, coordinated the design, data analysis and contributed to the writing of this paper.

Ahmad Kamil Mahmood: Led and participated in all experiments, focus on software agent's design studies and the personal monitoring system investigation for hazardous field. He also has contributed to the writing and revision of the manuscript.

Jafreezal Jaafar: Participated in all experiments, focus on the application of fuzzy logic and contributed to the writing and the revision of this paper.

\section{Ethics}

For further clarification, justification and latest development progress in this research study, readers are invited to contact the authors directly. Misuse of any data in this paper is not under author's responsibility.

\section{References}

Bustince, H., F. Herrera and J. Montero, 2007. Fuzzy Sets and Their Extensions: Representation, Aggregation and Models. 1st Edn., Springer Verlag, Berlin, ISBN-10: 35407372279783540737223 , pp: 694.

Castillo, O. and P. Melin, 2008. Type-2 Fuzzy Logic: Theory and Applications. 1st Edn., Springer, Berlin, ISBN-10: 3540762833, pp: 244.

Khuen, C.W., C.H. Yong and F. Haron, 2005. A framework for multi-agent negotiation system using adaptive fuzzy logic in resource allocation. Int. J. Inform. Technol., 11: 35-49.

Padgham, L. and M. Winikoff, 2005. Developing Intelligent Agent System. 1st Edn., John Wiley and Sons, Chichester, ISBN-10: 0470861215, pp: 240. 
Padgham, L., J. Thangarajah and M. Winikoff, 2005. Tool support for agent development using the prometheus methodology. Proceedings of the 5th International Conference on Quality Software, Sept. 19-20, IEEE Xplore Press, pp: 383-388. DOI: 10.1109/QSIC.2005.66

Nwana, H.S. and D.T. Ndumu, 1997. An Introduction to Agent Technology. In: Software Agents and Soft Computing Towards Enhancing Machine Intelligence, Hyacinth S. Nwana and Nader Azarmi (Eds.), Springer, Berlin Heidelberg, ISBN: 978-3540-62560-5, pp: 1-26.
Paolucci, M. and R. Sacile, 2005. Agent-Based Manufacturing and Control Systems. 1st Edn., CRC Press, Boca Raton, ISBN-10: 1574443364, pp: 269.

Wooldridge, M., 2009. An Introduction to MultiAgent Systems. 2nd Edn., John Wiley and Sons, Chichester, ISBN-10: 0470519460, pp: 484. 